\title{
Marco A. López, a Pioneer of Continuous Optimization in Spain
}

\section{Maria Josefa Cánovas ${ }^{1} \cdot$ Alexander Kruger $^{2} \cdot$ Hoang Xuan Phu $^{3} \cdot$ Michel Théra $^{2,4}$ (D}

Received: 25 November 2019 / Accepted: 26 November 2019 / Published online: 5 March 2020

(C) Vietnam Academy of Science and Technology (VAST) and Springer Nature Singapore Pte Ltd. 2020

\section{Marco's Research Path}

Marco Antonio López Cerdá was born on August 1, 1949 and grew up in Alcoy, province of Alicante, Spain. He graduated from the University of Valencia in 1971 and got his $\mathrm{PhD}$ degree in 1973 under the supervision of Professor Segundo Gutiérrez Cabria. He joined the University of Valencia as Associate Professor in 1978, became Full Professor in 1981, and moved to University of Alicante in 1985 as a Professor of Statistics and Operations Research, where he contributed tremendously to the development of a research group in optimization and related topics. Since September 2019 he is Emeritus Professor of this university. Marco López is Doctor Honoris Causa at the University of Limoges (since 2012) and Adjunct Professor of the Centre for Informatics and Applied Optimization (CIAO) at Federation University Australia (since 2013). In 2013 he was elected a Corresponding Member of the Spanish Royal Academy of Sciences.

Marco López has been a teacher for several generations of Spanish mathematicians, and adviser or co-adviser of $18 \mathrm{PhD}$ students in several branches of Operations Research (OR) such as semi-infinite programming, network flows, assignment problems, convex

Michel Théra

michel.thera@unilim.fr

Maria Josefa Cánovas

canovas@umh.es

Alexander Kruger

a.kruger@federation.edu.au

Hoang Xuan Phu

hxphu@math.ac.vn

1 Center of Operations Research, Miguel Hernández University of Elche, 03202 Elche (Alicante), Spain

2 Centre for Informatics and Applied Optimization, Federation University Australia, POB 663, Ballarat, VIC 3350, Australia

3 Institute of Mathematics, Vietnam Academy of Science and Technology, Hanoi, Vietnam

4 XLIM UMR-CNRS 7252 Université de Limoges, Limoges, France 
optimization, graphs, scheduling, game theory, and variational analysis. He has been the leader of many national and international research projects, both theoretical (basic research) and applied (for the university community services, the city and province of Alicante, and bank branches networks).

Marco has written several books. His book Linear Semi-Infinite Optimization [33], coauthored with Miguel Ángel Goberna and published by John Wiley \& Sons in 1998, has become a classical monograph in the field.

Marco's research activity has been increasing with time: out of his 146 publications appearing in MathSciNet more than 60 papers have been published in the last decade. These are mostly papers in top journals like, for instance, Mathematical Programming, Mathematics of Operations Research, SIAM Journal on Optimization, Journal of Optimization Theory and Applications, European Journal of Operational Research, Optimization. Marco's collaborative ability is remarkable. The list of his co-authors includes 60 names.

Pioneer among Spanish mathematicians of international caliber in the field of operations research, Marco jointly with his PhD student at that time Enriqueta Vercher were the first Spanish authors to publish in Mathematical Programming in 1983 (see [44]).

We want to point out Marco's service to the OR community. He served as co-editor-inchief of TOP (the OR journal of SEIO, the Spanish Society of Statistics and Operations Research) from 2000 to 2007. He serves as Associate Editor of several international journals, including International Abstracts of Operations Research, Journal of Industrial and Management Optimization, Journal of Optimization Theory and Applications, Set-Valued and Variational Analysis, RACSAM, etc. Marco was elected vice-president of SEIO in 1985 and 1998, elected president of SEIO in 1986, and served as chair of the Managing Board of EUROPT, the EURO Working Group on Continuous Optimization, since 2008 to 2010. He has been organizer or co-organizer of several important national and international conferences.

From July 2008 to April 2012 Marco López was the coordinator of i-MATH Consolider, a huge project of the Spanish Ministry of Education for all areas of mathematics, gathering more than 300 research groups, and with a budget of 7.5 million euros.

Marco has enormous general knowledge and is a very versatile person. He is a strong advocate for improving the quality of education and mathematics research at Spanish universities, and promoting the advantages of research for the society in general.

We would like to conclude this part by saying that beside every great man, there is an even greater woman, and Marco (as he said several times) is the first to recognize the amazing contributions of his wife, Maria Pilar, in enabling a fulfilled academic life and family life with 2 children (Pili and Marco) and four grandchildren!

\section{Some Key Qualities of Marco as Observed by His Colleagues}

The following paragraphs quote verbatim the words of some colleagues. They were written - and gathered in an unpublished book of 2010 — with the aim of paying tribute to Marco on the occasion of his 60th birthday. Now, we take up these words to illustrate a selection of the key personal and professional attributes of Marco.

\section{- Knowledge of mathematics}

“... At this occasion, I further realized, but this time at a more personal level, the deepness of Marco's knowledge in mathematics which covers so many areas such as convex analysis, functional analysis, optimization from theoretical formalism to numerical methods, as well as applications... I truly appreciated his remarkable abilities, 
his enthusiasm for investigating new fields, and his sense of perfection, all qualities that are very stimulating for his collaborators." Alfred Auslender, Institut Camille Jordan, France.

\section{- Research capability}

“... We all know that Marco is an outstanding mathematician, an international leader in semi-infinite optimization and related topics, the founder of the strong Spanish research group in these areas. But only those who actually worked with him will share my feelings on how deeply he approaches mathematical problems trying to understand the very essence of the issue and how strongly he contributes in joint projects, from the point of view of both ideas and techniques.” Boris S. Mordukhovich, Wayne State University, USA.

- Undergraduate teaching skills

“... He was a genuine lord. Etched on my memory ... his clear handwriting and organization on the blackboard. When writing on it, he sometimes got further to see in perspective his writing, checking if what he wrote was clear; if something, even a minimal thing like the tail of the ' $a$ ' was not completely perfect, then he deleted it and wrote it again... I have never had another professor who knew so much from so many things... I must say that he has been the best teacher I have ever had." Isabel Molina, Universidad Carlos III de Madrid Spain.

- Hospitality

“... Of course, I benefited much from our scientific discussions and from your profound knowledge of many areas of mathematics. But today I like to emphasize some other side of your personality: your warm, perfect hospitality. I was several times your guest, and I was impressed and overwhelmed each time. To be your guest means to have a big cultural experience because of many, many impressions of your country and the interesting information on Spanish and local history and traditions. It also means a culinary highlight...” Diethard Klatte, University of Zurich, Switzerland.

- Training of young researchers

“... Another contribution which is not immediately seen in my point of view, at least by Internet, is the big work of Marco in forming scientific groups and the establishment of recognized centers... I had the opportunity (and the chance) to be one of his postdoctoral students, and I realized that together with the famous scientific personality, he is a very fine person with many wonderful qualities. A student not only learns theorems and corollaries from him but also the love of doing them. He is a very patient person and very gracious at the same time as a good listener..." Abderrahim Hantoute, Center for Mathematical Modeling, University of Chile.

- Leadership abilities

"... From the performance of Marco in the meeting we held in the Executive Council of $i$-Math ${ }^{1}$, I highlight his good character, his calmness, his ability to negotiate and reach agreements, and his dedication to the job.” Eduardo Casas, University of Cantabria, Spain.

- Thoroughness in writing and reviewing

"The first time that I have been in contact with the mathematical opus of Marco was probably several years ago. I was one of the referees of a paper he submitted to a journal. Then, I understood that I was facing one of the best specialists in the field of linear or convex semi-infinite programming and well posedness problems... Also, I have

\footnotetext{
${ }^{1}$ It refers to the period when Marco was the coordinator of the huge Spanish project i-Math Consolider.
} 
asked Marco to act as a referee for some papers that I was handling, the job was done in due time, carefully, with appreciated helpful comments for the authors. I consider Marco as a very good and deep mathematician.” Jean-Pierre Crouzeix, Blaise Pascal University, France.

- Scientific benchmark

"... I met Marco in the mid-nineties. We were at a conference... I was curious about the reasons a senior (senior, not old) man could have to be one of the few persons in the audience. Not only that: since he discussed with the speakers after their talks, I realized he was even paying attention! ... Enthusiasm for learning and teaching maths, scientific thoroughness, high estimation of the scientific work of others, and unbounded working capacities are qualities I mentally linked to his face during this conference. Time has given me the chance of meeting Marco much closer... Saying that he is my scientific benchmark in OR in Spain is an accurate first-order approximation to what I do feel." Emilio Carrizosa, University of Seville, Spain.

- Internationalization of the Spanish research

“... I still remember my first SEIO meeting in 1988... In those days, research was not very popular within the Spanish OR community but since the very beginning my generation realized that things were about to change and we fixed our scientific benchmark in a few relevant researchers that had gained respect for their doubtless trajectory and excellence... There are a few names that would deserve to be mentioned but in this occasion I shall only mention professor López-Cerda, our friend Marco... Marco has been one of the main responsible persons for the current international character of TOP, the Spanish OR journal.” Justo Puerto, University of Seville, Spain.

- Sense of humor

"... Moreover, Marco is an outstanding speaker, not only when he speaks of mathematics. It is impossible to be bored when he is around, he has a contagious sense of humor and has a special ability to mix it with work, with people, with life." Ignacio García Jurado, Coruna University, Spain.

\section{Research Contributions}

Semi-Infinite Programming The main research interests and contributions of Marco López have been in the area of semi-infinite programming (SIP). The first series of papers coauthored with Miguel Ángel Goberna, Jesus Pastor and Enriqueta Vercher, in the period 1980-2000, dealt with the geometry of semi-infinite linear inequality systems, as well as with the the theory of linear semi-infinite optimization problems, for the primal and the dual problem (in the sense of Haar). A special attention was paid to deriving semi-infinite versions of theorems of alternative (by Farkas, Gale, Gordan, Motzkin, etc.). Topics like consistency, redundancy, discretizability and reducibility, which are essential for the numerical approach to solving linear SIP problems, were extensively studied. The results obtained in these papers, most of which are co-authored with Miguel Ángel Goberna, were included in the monograph Linear Semi-Infinite Optimization [33]. Among these contributions, we highlight below some papers which seem to us to be the most relevant. The choice is very subjective.

- In [31], by means of an appropriate use of the subdifferential mapping, the connections among duality theory, solvability properties, and convergence properties of sequences of discretized linear semi-infinite programs were made explicit. 
- In [32], the behavior of consistent semi-infinite linear inequality systems was analyzed with respect to many features of the solution set such as boundedness, dimension, description of its boundary, redundant inequalities, minimality, finite reduction, etc.

- Duality in linear SIP, and its relationship with discretizability and reducibility, was deeply studied in [34], where it was proved that the Farkas-Minkowski property is crucial for the so-called uniform duality.

- In [29], the powerful tool of the Farkas-Minkowski constraint qualification is exploited in the framework of convex SIP, exploring its relationship with stronger qualification conditions.

- Infinite-dimensional versions of the Farkas-Minkowski constraint qualification were established, and their role in the optimality conditions in convex optimization was clarified in [28] and [30].

Algorithms Marco López has made significant contributions to the algorithmic solution of SIP problems. We comment on the most relevant ones.

- In [1], two simplex-like methods are proposed for solving linear SIP's whose feasible regions are quasi-polyhedral sets, that is, sets whose intersection with an arbitrary polytope is a polytope.

- In [48], an exchange-type method for convex SIP is proposed with the feature that no global optimization needs to be carried out at each iteration to detect the (almost) most violated constraints.

- In [1], the authors formulate the strong separation of two sets in a normed space as an infinite-dimensional optimization problem, and give a method based on an approximation scheme which solves a SIP problem in each iteration without requiring any global optimization.

- In [4], penalty and smoothing methods (of Remez-type) for solving min-max convex SIP problems are proposed and their convergence is proved, whereas in [5] some computational estimates with these methods, for comparison purposes, is provided.

- In [2], some quasi-Newton methods to solve generalized equations in Banach spaces are examined under metric regularity.

Stability in SIP Starting from 1995, mainly in collaboration with Maria Josefa Cánovas, Miguel Ángel Goberna, Juan Parra, Maxim Todorov, Francisco Javier Toledo, and Virginia Vera de Serio, Marco López has published a series of papers on qualitative stability in SIP (linear and convex), whose general aim is to provide conditions under which sufficiently small perturbations of the nominal problem provoke small changes in the optimal value, the optimal set and the feasible set. The stability notions involved in this analysis are lower and upper semi-continuity (in Berge and Hausdorff senses), ill-posedness, etc.

- In [37], the Berge lower-semicontinuity of the feasible set mapping in linear SIP is completely characterized.

- In [38], the upper-semicontinuity of the feasible set mapping is studied; its complete characterization is given in [11] through the notion of reinforced system.

- In [12], the lower/upper semicontinuity of the optimal set mapping and the wellposedness property (in the sense of Hadamard) are characterized for linear SIP's, whereas these results are extended to the convex setting in [43].

- In [15] and [17], the ill-posedness in linear SIP with respect to various properties such as consistency, solvability, etc. (i.e., the boundary separating problems enjoying such a property and problems for which the property fails) is analyzed in detail. 
- Qualitative stability when only some of the coefficients are perturbed and when the mappings are restricted to their domains, is the main subject of [24] and [25]. Infinitedimensional counterparts of this qualitative analysis are performed in [26] and [27].

Lipschitz-type Stability Properties Also known as quantitative stability, as these properties aim to measure the level of stability of the mappings of interest (feasible set, optimal set, and optimal value mappings). These properties have important consequences in the overall stability of SIP, and affects also the numerical complexity of algorithms. The properties studied by Marco López and his co-authors are, mainly, pseudo-Lipschitzness, calmness, and distance to ill-posedness. Special attention is paid to characterizing, or estimating, the corresponding moduli as they quantify the level to which such a stability property holds.

- In [6, 7, 9, 19] (co-authored with M.J. Cánovas, A. Dontchev, A. Hantoute, D. Klatte, B. Mordukhovich, and J. Parra), the pseudo-Lipschitz property of the feasible and the optimal set mappings (or metric regularity of the inverse mappings), both in the linear and in convex settings, are approached; the last one is developed in infinite-dimensional spaces exploiting the properties of coderivatives, as it is also done in [41].

- In the field of calmness, the main contributions of Marco López (jointly with M.J. Cánovas, R. Henrion, A. Kruger, J. Parra and M. Théra) are as follows: an expression for the calmness modulus of the mapping in linear programming under canonical perturbations (objective function and right-hand side of the constraints), involving limits of subdifferentials [10]; left-hand-side perturbations of the constraints system added into the analysis in [18]; and the outer limits of subdifferentials of max-functions and calmness moduli for feasible and optimal set mappings dealt with in [8].

- In $[13,14,16]$, the distance to ill-posedness (in terms of the distance to infeasibility and to unsolvability) is studied.

Subdifferential Calculus The subdifferential of the supremum of convex functions is a key tool in convex analysis. In fact, Fritz-John and KKT-type optimality conditions for (semiinfinite) convex optimization can be approached by appealing to the subdifferential of the supremum of the constraint functions.

- In [39] (co-authored with Hantoute and Zălinescu), the first general formula for the subdifferential of the supremum function was established.

- Later on, in a series of papers co-authored with R. Correa and A. Hantoute [20-23], various formulas for the subdifferential of the supremum were established, extending and improving some classical results (due to Brøndsted, Valadier, Volle, etc.) as some standard continuity assumptions are dropped or weakened. In the last two quoted papers, Fritz-John and KKT optimality conditions for convex SIP are derived under very weak assumptions by applying the obtained subdifferential rules.

Game Theory The (unpublished) doctoral thesis of Marco López was on cooperative game theory, and it focused on Markov processes of cooperation and the valuation of a game. During most of his career, Marco has been fond of the game theory and delivered many courses and seminars on this topic. We quote some of his contributions.

- In [45], a generalization of the two-person zero-sum semi-infinite game introduced by A. L. Soyster (Management Sci. 21, 806-812, 1974/75) is provided. The pure strategies for player I are picked from an infinite family of convex functions, whereas the set of mixed strategies available to player II is a closed convex set. 
- The paper [40] formulates a generalization of the model of M. Balch (Differential games and related topics, Proc. Int. Summer School, Varenna, 1970, North-Holland, Amsterdam, 1971) and analyzes the oligopoly (and the Nash-Cournot and Pareto equilibria) from the point of view of differential games theory.

- In [47], the benefit sharing in holding situations is modeled as a big boss game, and its most relevant properties are examined.

- Finally, in [46] the authors prove that the transportation games have nonempty cores, and an LP-procedure describing this solution is shown.

Miscellaneous Marco López has contributed to many other areas which include: robustness, infinite-dimensional duality theory, stationarity and regularity, error bounds, and Hölder stability.

Marco has published several surveys on SIP. The most cited one is [42] (co-authored with G. Still), whereas the most recent survey [36] reviews the state of the art in the theory of deterministic and uncertain linear semi-infinite programs, presents some numerical approaches to this type of problems, and describes a selection of recent applications in a variety of fields. Recently Marco López has published another two books: monograph Post-Optimal Analysis in Linear Semi-Infinite Optimization [35] with M.A. Goberna, and Nonlinear Optimization [3] with F.J. Aragón-Artacho, M.A. Goberna and M.M.L. Rodríguez. The last one is a great companion for upper level undergraduate students in optimization.

\section{References}

1. Anderson, E.J., Goberna, M.A., López, M.A.: Simplex-like trajectories on quasi-polyhedral convex sets. Math. Oper. Res. 26, 147-162 (2001)

2. Aragón-Artacho, F.J., Belyakov, A., Dontchev, A.L., López, M.A.: Local convergence of quasi-Newton methods under metric regularity. Comput. Optim. Appl. 58, 225-247 (2014)

3. Aragón-Artacho, F.J., Goberna, M.A., López, M.A., Rodríguez, M.L.: Nonlinear Optimization. Springer Undergraduate Texts in Mathematics and Technology. Springer, Cham (2019)

4. Auslender, A., Goberna, M.A., López, M.A.: Penalty and smoothing methods for convex semi-infinite programming. Math. Oper. Res. 34, 303-319 (2009)

5. Auslender, A., Ferrer, A., Goberna, M.A., López, M.A.: Comparative study of RPSALG algorithm for convex semi-infinite programming. Comput. Optim. Appl. 60, 59-87 (2015)

6. Cánovas, M.J., Dontchev, A.L., López, M.A., Parra, J.: Metric regularity of semi-infinite constraint systems. Math. Program. Ser. B 104, 329-346 (2005)

7. Cánovas, M.J., Hantoute, A., López, M.A., Parra, J.: Stability of indices in the KKT conditions and metric regularity in convex semi-infinite optimization. J. Optim. Theory Appl. 139, 485-500 (2008)

8. Cánovas, M.J., Henrion, R., López, M.A., Parra, J.: Outer limit of subdifferentials and calmness moduli in linear and nonlinear programming. J. Optim. Theory Appl. 169, 925-952 (2016)

9. Cánovas, M.J., Klatte, D., López, M.A., Parra, J.: Metric regularity in convex semi-infinite optimization under canonical perturbations. SIAM J. Optim. 18, 717-732 (2007)

10. Cánovas, M.J., Kruger, A.Y., López, M.A., Parra, J., Théra, M.: Calmness modulus of linear semi-infinite programs. SIAM J. Optim. 24, 29-48 (2014)

11. Cánovas, M.J., López, M.A., Parra, J.: Upper semicontinuity of the feasible set mapping for linear inequality systems. Set-Valued Anal. 10, 361-378 (2002)

12. Cánovas, M.J., López, M.A., Parra, J., Todorov, M.I.: Stability and well-posedness in linear semi-infinite programming. SIAM J. Optim. 10, 82-89 (1999)

13. Cánovas, M.J., López, M.A., Parra, J., Toledo, F.J.: Distance to ill-posedness and the consistency value of linear semi-infinite inequality systems. Math. Program. Ser. B 103, 95-126 (2005)

14. Cánovas, M.J., López, M.A., Parra, J., Toledo, F.J.: Distance to solvability/unsolvability in linear optimization. SIAM J. Optim. 16, 629-649 (2006) 
15. Cánovas, M.J., López, M.A., Parra, J., Toledo, F.J.: Ill-posedness with respect to the solvability in linear optimization. Linear Algebra Appl. 416, 520-540 (2006)

16. Cánovas, M.J., López, M.A., Parra, J., Toledo, F.J.: Distance to ill-posedness in linear optimization via the Fenchel-Legendre conjugate. J. Optim. Theory Appl. 130, 173-183 (2006)

17. Cánovas, M.J., López, M.A., Parra, J., Toledo, F.J.: Sufficient conditions for total ill-posedness in linear semi-infinite optimization. Eur. J. Oper. Res. 181, 1126-1136 (2007)

18. Cánovas, M.J., López, M.A., Parra, J., Toledo, F.J.: Calmness of the feasible set mapping for linear inequality systems. Set-valued Var Anal. 22, 375-389 (2014)

19. Cánovas, M.J., López, M.A., Mordukhovich, B.S., Parra, J.: Variational analysis in semi-infinite and infinite programming, I: Stability of linear inequality systems of feasible solutions. SIAM J. Optim. 20, $1504-1526$ (2009)

20. Correa, R., Hantoute, A., López, M.A.: Towards supremum-sum subdifferential calculus free of qualification conditions. SIAM J. Optim. 26, 2219-2234 (2016)

21. Correa, R., Hantoute, A., López, M.A.: Valadier-like formulas for the supremum function I. J. Convex Anal. 25, 1253-1278 (2018)

22. Correa, R., Hantoute, A., López, M.A.: Valadier-like formulas for the supremum function II: The compactly indexed case. J. Convex Anal. 26, 299-324 (2019)

23. Correa, R., Hantoute, A., López, M.A.: Moreau-rockafellar-type formulas for the subdifferential of the supremum function. SIAM J. Optim. 29, 1106-1130 (2019)

24. Daniilidis, A., Goberna, M.A., López, M.A., Lucchetti, R.: Lower semicontinuity of the feasible set mapping of linear systems relative to their domains. Set-valued Var Anal. 21, 67-92 (2013)

25. Daniilidis, A., Goberna, M.A., López, M.A., Lucchetti, R.: Stability in linear optimization under perturbations of the left-hand side coefficients. Set-valued Var Anal. 23, 737-758 (2015)

26. Dinh, N., Goberna, M.A., López, M.A.: On the stability of the feasible set in optimization problems. SIAM J. Optim. 20, 2254-2280 (2010)

27. Dinh, N., Goberna, M.A., López, M.A.: On the stability of the optimal value and the optimal set in optimization problems. J. Convex Anal. 19, 927-953 (2012)

28. Dinh, N., Goberna, M.A., López, M.A., Son, T.Q.: New Farkas-type constraint qualifications in convex infinite programming. ESAIM Control Optim. Calc. Var. 13, 580-597 (2007)

29. Fajardo, M.D., López, M.A.: Locally Farkas-Minkowski systems in convex semi-infinite programming. J. Optim. Theory Appl. 103, 313-335 (1999)

30. Goberna, M.A., Jeyakumar, V., López, M.A.: Necessary and sufficient constraint qualifications for solvability of systems of infinite convex inequalities. Nonlinear Anal. 68, 1184-1194 (2008)

31. Goberna, M.A., López, M.A.: Optimal value function in semi-infinite programming. J. Optim. Theory Appl. 59, 261-279 (1988)

32. Goberna, M.A., López, M.A.: A theory of linear inequality systems. Linear Algebra Appl. 106, 77-115 (1988)

33. Goberna, M.A., López, M.A.: Linear Semi-Infinite Optimization. Wiley, Chichester (1998)

34. Goberna, M.A., López, M.A.: On duality in semi-infinite programming and existence theorems for linear inequalities. J. Math. Anal. Appl. 230, 173-192 (1999)

35. Goberna, M.A., López, M.A.: Post-Optimal Analysis in Linear Semi-Infinite Optimization. Springer, New York (2014)

36. Goberna, M.A., López, M.A.: Recent contributions to linear semi-infinite optimization: an update. Ann Oper. Res. 271, 237-278 (2018)

37. Goberna, M.A., López, M.A., Todorov, M.I.: Stability theory for linear inequality systems. SIAM J. Matrix Anal. Appl. 17, 730-743 (1996)

38. Goberna, M.A., López, M.A., Todorov, M.I.: Stability theory for linear inequality systems II: Upper semicontinuity of the solution set mapping. SIAM J. Optim. 7, 1138-1151 (1997)

39. Hantoute, A., López, M.A., Zălinescu, C.: Subdifferential calculus rules in convex analysis: a unifying approach via pointwise supremum functions. SIAM J. Optim. 19, 863-882 (2008)

40. López, M.A.: Dynamic strategic models for markets with fixed prices. (Spanish) Trab. Estad. Investig. Oper. 29, 40-66 (1978)

41. López, M.A., Ridolfi, A.B., Vera de Serio, V.N.: On coderivatives and Lipschitzian properties of the dual pair in optimization. Nonlinear Anal. 75, 1461-1482 (2012)

42. López, M.A., Still, G.: Semi-infinite programming. Eur. J. Oper. Res. 180, 491-518 (2007)

43. López, M.A., Vera de Serio, V.N.: Stability of the feasible set mapping in convex semi-infinite programming. In: Boberna, M.A., López, M.A. (eds.) Semi-Infinite Programming. Nonconvex Optimization and Its Applications, vol. 57, pp. 101-120. Kluwer Academic Publishers, Dordrecht (2001)

44. López, M.A., Vercher, E.: Optimality conditions for nondifferentiable convex semi-infinite programming. Math. Program. Ser. A 27, 307-319 (1983) 
45. López, M.A., Vercher, D.E.: Convex semi-infinite games. J. Optim. Theory Appl. 50, 289-312 (1986)

46. Sánchez-Soriano, J., López, M.A., García-jurado, I.: On the core of transportation games. Math. Soc. Sci. 41, 215-225 (2001)

47. Tijs, S., Meca, A., López, M.A.: Benefit sharing in holding situations. Eur. J. Oper. Res. 162, 251-269 (2005)

48. Zhang, L., Wu, S.-Y., López, M.A.: A new exchange method for convex semi-infinite programming. SIAM J. Optim. 20, 2959-2977 (2010)

Publisher's Note Springer Nature remains neutral with regard to jurisdictional claims in published maps and institutional affiliations. 